der Straße“. Ungeachtet von Meinungsumfragen war und bleibt Sicherheitspolitik eine Domäne der Eliten. Das sicherheitspolitische Bewusstsein muss also vor allem dort geschaffen werden, wo Sicherheitspolitik gemacht bzw. wo darüber entschieden wird - nämlich in den einschlägigen Ministerien und vor allem im Parlament. Dort entscheidet sich die Zukunfts- und Bündnisfähigkeit Deutschlands. Gefragt ist ein sicherheitspo- litisches Selbstbewusstsein der politischen Klasse in Deutschland, das gefestigt genug ist, um wichtige Entscheidungen parteiunabhängig und ohne das permanente Schielen auf die Demoskopie zu treffen. Angesichts der Aufgabe, einen solchen Bewusstseinswandel herbeizuführen, erscheinen die Herausforderungen in Afghanistan fast schon wieder einfach.

\title{
Das internationale Engagement in Afghanistan: Plädoyer für einen umfassenden Strategiewechsel
}

\author{
Hans-Georg Ehrhart*/Roland Kaestner**
}

\begin{abstract}
The Afghan reality and the activities of the international community are diverging more and more. However, NATO's vision for Afghanistan, as adopted at the Bucharest summit, basically sticks to the long-standing assessment of the situation and the related strategy. The following analysis concentrates on the actual state of affairs and structural parameters in Afghanistan. While not denying the relevance of regional factors of influence it is argued that the resolution of the conflict primarily depends on internal Afghan developments. Given the present state of affairs and the mid- and long-term development trends of this country, the core conclusion of this article is that there is an urgent need for a comprehensive strategy change.
\end{abstract}

Keywords: Afghanistan: strategy, conflict, internal development, options, Afghanistan, Strategie, Konflikt, interne Entwicklung, Optionen

\section{Einleitung ${ }^{1}$}

$\mathrm{N}$ ach den Terroranschlägen vom 11. September 2001 wurden Al-Qaida und das Taliban-Regime durch eine von den USA geführte Koalition der Willigen (Operation „Enduring Freedom“) aus Afghanistan vertrieben. Seitdem engagiert sich die internationale Staatengemeinschaft in diesem Land. Deutschland nimmt dabei von Beginn an eine herausgehobene Rolle ein. Auf dem Petersberg bei Bonn wurde der Friedens- und Wiederaufbauprozess Afghanistans eingeleitet („Bonn process“). Dort beschlossen die Vertreter der siegreichen afghanischen Fraktionen der Nordallianz, der Vereinten Nationen, der USA und anderer internationaler Akteure, Afghanistan zu einer marktwirtschaftlichen Demokratie zu entwickeln. ${ }^{2}$ Die Internationalen Sicherheitsassistenzkräfte (ISAF) sollten zu einem sicheren Umfeld beitragen und den Aufbau afghanischer Sicherheitskräfte unterstützen. Vier Jahre später wurde der Bonn Process abgelöst durch den Afghanistan Compact

Dr. Hans-Georg Ehrhart ist Leiter des Zentrums für Europäische Friedens- und ** Sicherheitsstudien (ZEUS) am IFSH.

Oberst i.G. Roland Kaestner ist Dozent für Strategie an der Führungsakademie der Bundeswehr in Hamburg.

1 Dieser Beitrag wurde referiert (double blind peer-review).

2 Bonn Agreement: Agreement on provisional arrangements in Afghanistan pending the re-establishment of permanent government institutions, Bonn, 5 . Dezember 2002.
$(\mathrm{AC})^{3}$. Dieser beschreibt eine bis Ende 2010 angelegte komplexe Strategie, die aus den drei Säulen Sicherheit, Governance und Entwicklung besteht. Sie bildet die Grundlage für den im selben Jahr verabschiedeten vorläufigen nationalen Entwicklungsplan der afghanischen Regierung. Nach ersten Fortschritten verschlechterte sich die Lage seit 2005 kontinuierlich. Die Entwicklung der afghanischen Realität und die Maßnahmen der internationalen Gemeinschaft klaffen zunehmend auseinander. Damit wird eine Überprüfung sowohl der Lagefeststellung als auch der Strategie dringend erforderlich, zumal die auf dem Bukarester NATO-Gipfel verabschiedete Vision für Afghanistan an der alten Lagebeurteilung und dem strategischen Konzept grundsätzlich festhält ${ }^{4}$.

Die folgende Analyse konzentriert sich auf die Lage und strukturelle Entwicklungsparameter in Afghanistan. Regionale Einflussfaktoren sollen dadurch nicht negiert werden. Doch hängt eine Befriedung des Konflikts primär von der Entwicklung im Lande selbst ab. Die gegenwärtige Lage und die mittel- und langfristigen Entwicklungstrends in Afghanistan zeigen, so die Kernfolgerung dieses Beitrags, dass ein umfassender Strategiewechsel notwendig ist.

3 The Afghanistan Compact, London, 31. Januar - 1. Februar 2006, unter: http://www.unama-afg.org/news/_londonConf/_docs/06jan30-AfghanistanCompact-Final.pdf (Zugriff: 28. April 2008).

4 NATO: ISAF's Strategic Vision. Declaration by the Heads of State and Government of the Nations Contributing to the UN-mandated NATO-led International Security Assistance Force (ISAF) in Afghanistan, 3. April 2008. 


\section{Die Lage in Afghanistan}

Sicherheit: Die Sicherheitslage hat sich seit 2005 verschlechtert. Die Zahl der sicherheitsrelevanten Ereignisse steigt von Jahr zu Jahr an. Die Zwischenfälle stiegen von ca. 3.100 im Jahre 2005 auf ca. 8.000 in 2006 und sind nach offiziellen US-Angaben in den Jahren 2007 und 2008 nochmals um je 30 Prozent angewachsen. ${ }^{5}$ Insbesondere die Zahl der Selbstmordattentate - ein bis vor Kurzem noch unbekanntes Phänomen in Afghanistan - nahm zu. Wurden 200517 Selbstmordattentate begangen, waren es 123 im Jahr 2006 und 131 ein Jahr darauf. Die sogenannten Feindelemente oder Neotaliban - dazu können islamistische oder nationalistische Paschtunen, Drogenhändler, lokale Kommandeure, „warlords“, Al-Qaida-Terroristen, ausländische Jihadisten, religiöse Fundamentalisten, Antizentralisten und autonome Kräfte zählen -, kontrollieren oder infiltrieren immer weitere Gebiete. Nach Schätzungen von Senlis stehen 54 Prozent des afghanischen Territoriums unter ihrer Kontrolle, in weiteren 38 Prozent sind sie partiell präsent. ${ }^{6}$ Nach anderen Quellen ist „nur“ ein Drittel des Landes als Hochrisikozone einzuschätzen. ${ }^{7}$ Mit diesem zunehmenden Einfluss korrespondiert die steigende Zahl der Luftangriffe durch ISAF (International Security Assistance Force) und OEF (Operation Enduring Freedom), die weit über entsprechende Aktivitäten im Irak hinausgeht, ebenso wie der Anstieg der gefallenen westlichen Soldaten ${ }^{8}$. Die Neo-Taliban sind eine fluide, sich ständig verändernde Größe. Dies entspricht der Tradition afghanischer Akteure, im Eigeninteresse wechselnde Koalitionen einzugehen mit dem Ziel, die Macht der Regierung in Kabul zu beschränken und die lokalen Herrschaftsansprüche zu erhalten. Schetter weist darauf hin, dass das Wort Taliban bei der afghanischen Bevölkerung keine Personengruppe mehr bezeichnet, sondern mittlerweile eine Art Lifestyle geworden ist. ${ }^{9}$ Die politische Haltung in den regierungsfeindlichen Regionen ist geprägt durch anti-zentralistische, anti-moderne und anti-westliche Einstellungen und verbindet lokale mit militant-islamischen Vorstellungen. Der Kampf zwischen dem Autonomieanspruch der lokalen Akteure und dem Herrschaftsanspruch des Staates durchzieht im Übrigen den gesamten Prozess der Staatsentwicklung in Afghanistan. Bislang führte jeder Versuch einen Zentralstaat zu etablieren zum Krieg.

Der Aufbau der afghanischen Sicherheitskräfte, die laut Afghanistan Compact die Sicherheit der Bürger schützen sollen, hinkt weit hinter der Planung her. Die afghanische Armee (ANA) kann noch als vergleichsweise positives Beispiel angeführt werden, obwohl sie mit bislang ca. 30.000-33.000 einsatzbereiten Soldaten die bis Ende 2010 vorgesehene Stärke

5 Cordesman, Anthony H.: The Struggle for "Pashtunistan": The Afghan-Pakistan War, CSIS, Oktober 2007, unter: http://www.csis.org/media/csis/pubs/101607 pashtunistan.pdf (Zugriff: 29. April 2008) und ders.: Follow the Money: Why the US is Loosing the War in Afghanistan, CSIS, Oktober 2008.

6 Stumbling into Chaos: Afghanistan on the Brink, London: Senlis Afghanistan, November 2007, S. 28

7 Cordesman, Anthony H.: The Afghan-Pakistan War: Threat Developments, Washington: CSIS, 1. Februar 2008, S. 32.

8 Ebd., S. 22 und S. 25.

9 Schetter, Conrad: Talibanistan - Der Anti-Staat, in: Internationales Asienforum, Bd. 38, Nr. 3-4, Freiburg: Arnold-Bergstraesser-Institut für Kulturwissenschaftliche Forschung, 2007, S. 233-257. von 80.000 Einsatzkräften nicht erreichen wird. ${ }^{10}$ Sie wurde von Anfang an weniger als Armee des afghanischen Staates aufgebaut, die unabhängig zu kämpfen in der Lage ist, sondern eher als amerikanische Hilfstruppe. ${ }^{11}$ Der Aufbau einer afghanischen Polizei (ANP) muss als Fehlschlag eingestuft werden. Die Daten aus dem Innenministerium, das zu den korruptesten Einrichtungen im Lande gehören soll und dringend reformiert werden müsste, sind mit entsprechender Skepsis zu behandeln. Die offiziell finanzierten Dienstposten - je nach Quelle 50.00070.000 Mann - weichen vermutlich erheblich von den tatsächlich vorhandenen Polizeikräften ab. So sollen tatsächlich nur annähernd 50 Prozent der offiziell gemeldeten ANP existieren, während die Gehälter für die Phantompolizisten in die Taschen korrupter Akteure fließen. ${ }^{12}$ Erschwerend kommt hinzu, dass die meisten der Polizisten Analphabeten sind und die 25 an der Ausbildung beteiligten Staaten über kein abgestimmtes Ausbildungskonzept verfügen. Darüber hinaus häufen sich Meldungen, dass die Polizei selbst zum Sicherheitsproblem wird. ${ }^{13}$ Damit steht sowohl quantitativ wie qualitativ das gesetzte Ziel von 82.000 Polizisten bis Ende 2010 in Frage. Vor diesem Hintergrund ist es nicht verwunderlich, dass mittlerweile sowohl die NATO-geführte ISAF, die ursprünglich Stabilisierungsaufgaben wahrnehmen sollte, als auch die US-geführte Operation Enduring Freedom, die sich zunächst auf den Antiterrorkampf konzentrierte, zunehmend in eine Aufstandsbekämpfung (Counterinsurgency) verwickelt sind, die militärisch zu gewinnen sehr unterschiedlich eingeschätzt wird. Unstrittig ist jedoch, dass mit Fortdauer dieses Engagements ein immer höherer Preis - personell, finanziell, ethisch - für diese Art des militärischen Einsatzes zu entrichten ist. ${ }^{14}$

Governance: Der zweite Reformbereich umfasst die Sektoren Regierungsführung, Rechtsstaatlichkeit und Menschenrechte. Angespornt durch die ersten Erfolge beim Aufbau staatlicher Strukturen (Wahlen, Parlamente, Verfassung) setzte der Afghanistan Compact zu hochgesteckte Ziele. Die Erwartungen an einen schnellen Demokratisierungsprozess erwiesen sich als übertrieben, erste Fortschritte beim Aufbau staatlicher Institutionen wurden überschätzt, nicht zuletzt, weil die Möglichkeiten eines „social engeneering“ in einer stark traditionalen Gesellschaft falsch eingeschätzt wurden. ${ }^{15}$ Ein weiterer Grund dafür ist das sogenannte „statebuilding paradox“. Dieses besteht darin, dass die internationale Gemeinschaft einerseits Hilfe und Unterstützung leistet, andererseits dadurch aber die Abhängigkeit Afghanistans stärkt und es faktisch zu einem

10 Maass, Citha: The Implementation of the Afghan Compact: Problems and Prospects, in: Ehrhart, Hans-Georg / Pentland, Charles (Eds.): The Afghanistan Challenge: Hard Realities and Strategic Choices, Montreal/Kingston: McGillQueen's Press 2009 (in Vorbereitung). Mittlerweile kursieren Planungen, die Truppenstärke der afghanischen Armee bis 2012 auf 134.000 Mann zu erhöhen. Der beschleunigte Aufbau soll 17 Milliarden US-Dollar kosten. Vgl. Frankfurter Allgemeine Zeitung, 12. November 2008.

11 Giustozzi, Antonio: Auxiliary Force or National Army? Afghanistan's ANA and the Counter-Insurgency Effort, in: Small Wars and Insurgencies, Bd. 18, Nr. 1, S. 45-67, 2007.

12 Wilder, Andrew: Cops or Robbers? The Struggle to Reform the Afghan National Police, Kabul: Afghanistan Research and Evaluation Unit, Issues Paper Series, Juli 2007, S. 7

13 Ebd.; Reforming Afghanistan's Police, International Crisis Group: Asia Report, Nr. 138, 30. August 2007; Ibrahimi, Sayed Yaqub: Afghan police part of the problem, Institute for War \& Peace Reporting, 6. Juni 2006.

14 Van Creveld, Martin: On Future War, London: Brassey's, 1991.

15 Roy, Olivier: Afghanistan : la difficile reconstruction d'un Etat, Paris: Institut d'études de sécurité de l'Union européenne, Cahier de Chaillot, Dezember 2004. 
Klientelstaat macht. ${ }^{16}$ Fortschritte werden nur anhand bürokratischer Maßstäbe beurteilt ${ }^{17}$ und die Antwort auf die Frage, wie diese Ziele effektiv, d.h. mit positiven Auswirkungen auf die Bevölkerung umgesetzt werden, wird vor allem in Kabul durch ein Patronagesystem blockiert, dass Korruption begünstigt. ${ }^{18}$ Bemühungen zum Aufbau subnationaler GovernanceStrukturen leiden unter mangelndem Engagement, fehlender Koordinierung und der Fixierung auf kurzfristige Sicherheitsimperative. ${ }^{19}$ Das rapide sinkende Ansehen von Präsident Karzai ist eine Folge dieser Entwicklung, zu der die internationale Gebergemeinschaft mit ihren übertriebenen Zielen und ihrem unkoordinierten Vorgehen nicht unerheblich beigetragen hat. Afghanistan ist eines der korruptesten Länder der Welt. Daran hat auch die internationale Präsenz nichts geändert, ja sie hat sie durch das reichlich fließende Geld der Geberländer möglicherweise sogar verschärft. ${ }^{20}$ Nach Einschätzung eines Teils der Bevölkerung ist die Lage derzeit schlimmer als zu Zeiten der Taliban. ${ }^{21}$

Entwicklung: Afghanistan soll sich zu einer freien Markwirtschaft entwickeln, angetrieben durch den privaten Sektor. Dieser wirtschaftsliberale Ansatz begünstigt in einem Land, das ökonomisch am Boden liegt, über keinen funktionierenden öffentlichen Sektor verfügt und in dem Rechtssicherheit fehlt, in erster Linie nur wenige Privilegierte, die sich das neue System zunutze machen können. Die Lebensbedingungen der meisten Afghanen haben sich verschlechtert. Insbesondere in den Paschtunengebieten kommt Entwicklungshilfe so gut wie nicht an und das, was ankommt, wird von den Aufständischen schnell wieder zerstört. ${ }^{22}$ Entwicklungspolitisch problematisch sind des Weiteren auch andere Aspekte, wie der durch die internationale Präsenz ermöglichte Brain drain. Da beispielsweise ein Fahrer bei einer NGO das Vielfache eines Universitätsprofessors verdient, arbeiten viele dringend gebrauchte afghanische Experten in anderen Bereichen und stehen nicht für die Entwicklung des Landes zur Verfügung. Zu erwähnen ist auch die immense Kluft zwischen dem Reichtum der Vertreter der Internationalen Gemeinschaft und den afghanischen „Normalbürgern“, die ebenso zu Frustration führt wie die Bemühungen, eine afghanische Zivilgesellschaft nach westlichem Vorbild aufzubauen. ${ }^{23}$ Es ist unstrittig, dass statistisch das Pro-Kopf-Einkommen von 182 US-Dollar im Jahr (!) in 2002/03

16 Nixon, Hamish: Aiding the State? International Assistance and the Statebuilding Paradox in Afghanistan, Kabul: Afghanistan Research and Evaluation Unit, Briefing Paper Series, April 2007.

17 Siehe: Maass, Citha (2008).

18 Koczy, Ute/Unmüßig, Barbara: Illusionen und Realitäten: Der steinige Weg des (entwicklungs-)politischen Aufbaus, in: Zeitschrift für Afghanistankunde, Nr. 2, April 2008, S. 22-55.

19 Nixon, Hamish: Subnational State-Building in Afghanistan, Kabul: Afghanistan Research and Evaluation Unit, Synthesis Paper Series, April 2008.

20 McConnell, J. Michael: Annual Threat Assessment of the Director of National Intelligence for the Senate Select Committee on Intelligence, 5. Februar 2008, Washington, unter: http://intelligence.senate.gov/080205/mcconnell.pdf (Zugriff 7. August 2008); Revitalizing our Efforts, Rethinking our Strategies, Washington: Center for the Study of the Presidency, Afghan Study Group Report, 2. Aufl., 30. Januar 2008.

21 Adams, Brad, Open Letter from Human Rights Watch to the International Afghanistan Support Conference on June 12, 2008, Human Rights Watch, 10. Juni 2008.

22 Johnson, Thomas H.: On the Edge of the Big Muddy: The Taliban Resurgence in Afghanistan, in: Niklas Swanström (Ed.), Uppsala: Central Asia-Caucasus Institute \& Silk Road Studies Program, China and Eurasia Forum Quarterly, Bd. 5, Nr. 2, S. 93-129, Mai 2007, S. 122 und S. 127.

23 Siehe: Roy, Olivier (2004), S. 57f. auf 344 US-Dollar in 2006/07 gestiegen ist. ${ }^{24}$ Gleichwohl gehört Afghanistan zu den ärmsten und am wenigsten entwickelten Ländern der Welt, denn statistisch gesehen muss jeder Afghane von weniger als einem US-Dollar am Tag leben. Im Bildungsbereich sind zwar Fortschritte zu verbuchen, sie sind jedoch marginal, wenn man ihnen das schnelle Bevölkerungswachstum, die hohe Arbeitslosigkeit und die immer noch extrem hohe Analphabetenrate entgegenhält. Der florierendste und zugleich problematischste Wirtschaftsbereich ist der Drogenhandel. Hier sind kontinuierlich hohe Wachstumsraten zu verzeichnen, mit extrem negativen Auswirkungen auf das Funktionieren staatlicher Strukturen (Korruption), die Sicherheit der Bevölkerung (Gewaltkriminalität) und die Auseinandersetzung mit den Neo-Taliban (Finanzierung des Krieges).

\section{Entwicklungsfaktoren}

Bevölkerung: Die afghanische Bevölkerung ist trotz eines jahrzehntelangen Krieges von 8,1 Millionen Menschen (1950) auf über 30 Mio. (2005) gewachsen. Die UNO prognostiziert bis 2030 einen weiteren Anstieg auf ca. 58,9 Mio. ${ }^{25}$ Obwohl die Einwohnerzahl von Kabul rasant wächst, leben mehr als 75 Prozent der Afghanen auf dem Land. Nach UNO-Angaben soll der Anteil der Landbevölkerung 2030 noch mehr als 65 Prozent betragen. Kinder unter 14 Jahren machen mit einem Anteil von ca. 45 Prozent die afghanische Gesellschaft zu einer der jüngsten der Welt - Tendenz steigend. ${ }^{26}$ Dieser sogenannte „youth bulge" in Verbindung mit Arbeitslosigkeit und Armut setzt die aufzubauenden politischen Strukturen unter hohen Stress. Er wird auch die tribalen Strukturen überfordern und spricht für eine konfliktreiche Zukunft.

Ressourcen: Afghanistan verfügt nur über wenige Ressourcen und diese sind überwiegend in den Händen regionaler Warlords und regierungsfeindlicher Akteure. Nur zehn Prozent der Bevölkerung hat Zugang zu Elektrizität, überwiegend in Stadtregionen. Öffentliche Güter (Wasser/Gesundheit/Bildung/Verwaltung etc.) stehen nur einer begrenzten Zahl von Menschen zur Verfügung. Angesichts des Bevölkerungswachstums wird sich diese Situation eher noch verschärfen. Selbst von der Ressource Drogen haben nach Schätzungen des Entwicklungsprogramms der Vereinen Nationen (UNDP) nur ca. 15 Prozent der Bevölkerung einen „Nutzen“, wobei sich die Einnahmen auf wenige mächtige Akteure konzentrieren. ${ }^{27}$ Mit dem Profit aus dem Drogengeschäft finanzieren aber fast alle Parteien ihre Privatarmeen.

Kultur: Das Land ist durch große ethnische und sprachliche Vielfalt geprägt. ${ }^{28}$ Die Paschtunen sind die größte ethnische Gruppe (ca. 42 Prozent) mit einem gesamtafghanischen Führungsanspruch, der von anderen nicht generell geteilt wird und

24 Center for Policy and Human Development: Afghanistan Human Development Report, Bridging Modernity and Tradition, Rule of Law and the Search for Justice, Kabul, 2007.

25 UNPP (2006): World Population Prospects, unter: http://esa.un.org/unpp/ p2k0data.asp.

26 Ebd.; Central Intelligence Agency (CIA), Office of Public Affairs: The World Factbook, Washington, April 2008.

27 United Nations Office on Drugs and Crime (UNODC): 2008 World Drug Report, Wien 2008.

28 Siehe: Cordesman, Anthony H. (2008), S. 42. 
insbesondere während der Bürgerkriegsjahre bis zum Ende Taliban-Herrschaft durch den Konflikt mit der Nordallianz zum Ausdruck kam. Die Analphabetenrate der erwachsenen Bevölkerung liegt über 70 Prozent, die von Frauen dürfte eher bei 90 Prozent liegen ${ }^{29}$. Aufgrund der Bevölkerungsentwicklung und der sehr stark patriarchalisch geprägten Stammeskultur wird sich dieser Fakt vermutlich nur marginal in den nächsten Jahrzehnten ändern. Bemerkenswert ist, dass sich der Ende der 1970er Jahre ausgebrochene innerafghanische Konflikt (der 1979 mit dem Einmarsch sowjetischer Truppen eskalierte) mit der damaligen kommunistischen Zentralregierung in Kabul an der Frage der Schulpflicht von Mädchen auf dem Lande entzündete.

Gesellschaft: Es gibt keine moderne afghanische Gesellschaft, sondern allenfalls kleine Inseln von Modernität in einigen Städten. Die für die Menschen zuverlässigsten Strukturen sind die Familie, der Stamm, der Clan und lokale Gemeinschaften. Sie fordern aber gleichzeitig eine Unterwerfung des Individuums unter die Gemeinschaft, die eine ausgeprägte soziale Kontrolle ausübt und ein erhebliches Hemmnis für sozialen Wandel darstellt. Das Paschtunwali, das mit den Begriffen Ehre, Würde, Stolz, Kampf und Männlichkeit das Selbstverständnis bei Paschtunen und in abgewandelter Form bei anderen Ethnien prägt, bestimmt das Verhalten der Afghanen gegenüber Fremden. ${ }^{30}$ Das Paschtunwali ist ein nicht ausformulierter Kanon kultureller Regel und Normen, die durch Familie, Clan und Stamm weitergegeben werden. Dieser Kodex sorgt für Verhaltenssicherheit und die Verantwortung von Familie und Clan für die soziale Absicherung des Einzelnen. Ihn auch nur teilweise durch geschriebenes Recht - insbesondere aus der westlichen Welt - zu ersetzen, wird zu erheblichen Verunsicherungen führen, aber auch bei bestimmten Eliten als ein Angriff auf ihre Macht und damit auf Ehre und Würde verstanden. Dieser Wertekanon ist „an uncompromising social code so profoundly at odds with Western mores that its application constantly brings one up with a jolt". ${ }^{31}$ Die Antwort wird, solange die gesellschaftliche Veränderung nicht durch die Menschen selbst vollzogen wird, eine gewaltsame sein. Ohne sozialen Wandel ist allerdings eine moderne arbeitsteilige Gesellschaft, die die Bedürfnisse einer wachsenden Zahl von Menschen befriedigen muss, nicht denkbar.

Wirtschaft: Die afghanische Gesellschaft ist eine Nomadenund Agrargesellschaft. Als extrem armes Binnenland ist Afghanistan abhängig von Auslandshilfe und vom Außenhandel. Etwa 80 Prozent der Menschen arbeiten im Agrarsektor. Es gibt einen sehr kleinen Industriesektor, der sich auf begrenzte Mengen von Gas, Kohle und Kupfer stützt sowie vor allem auf die kleinindustrielle Produktion von Textilien, Möbeln, Zement, Dünger, Schuhen, Seife und Teppichen. Die Wachstumsrate des BIP von über sieben Prozent im Jahre 2007 muss vor dem

29 United Nations Development Programme (UNDP): Afghanistan National Human Development Report, Security with a Human Face: Challenges and Responsibilities, Kabul 2004.

30 Glatzer, Bernt: Zum Pashtunwali als ethnischem Selbstportrait, in: Best, Günter/ Kößler, Reinhart (Hg): Subjekte und Systeme: Soziologische und anthropologische Annäherungen. Festschrift für Christian Sigrist zum 65 Geburtstag, Frankfurt: IKO-Verlag, 2000, S. 93-102. Barfield, Thomas/Nojumi, Neamat/Their, J. Alexander:, The Clash of Two Goods State and Non-State Dispute Resolution in Afghanistan, New York: US Institute of Peace, 2006.

31 Allen zitiert in Johnson, Thomas H. (2007), S. 121.
Hintergrund des sehr niedrigen Ausgangsniveaus gesehen werden. Die Arbeitslosigkeit wird offiziell mit 40 Prozent angegeben $^{32}$. Inoffiziell wird landesweit von einer Arbeitslosigkeit von ca. 70 Prozent im Süden und sogar von 90 Prozent im Osten gesprochen $^{33}$. Die beiden Wirtschaftssektoren, die permanent wachsen, sind die Drogenökonomie und der Transportsektor. Der erste Bereich macht ein Drittel der gesamten Wirtschaftsaktivitäten des Landes aus. ${ }^{34}$ Er wuchs mit einer Produktion von 3.400 Tonnen im Jahr 2002 auf 8.200 Tonnen in 2007, während die Anbaufläche im gleichen Zeitraum von 74.000 auf über 200.000 Hektar anstieg. ${ }^{35}$ Der andere prosperierende Sektor wird von der Transportmafia beherrscht. Diese gehört zu einem weltumspannenden System organisierter Kriminalität, das Schmuggelgüter aus, nach und durch Afghanistan befördert. ${ }^{36}$ Beide Sektoren nutzen eine mittlerweile globalisierte Stammesgesellschaft, ${ }^{37}$ die sich aus der wirtschafts- und kriegsbedingten Abwanderung mehrerer Millionen Afghanen seit den 1980er Jahren entwickelt hat. Sie bildet ein von Südasien über den Mittleren Osten bis nach Zentralasien reichendes Netzwerk, das für die Versorgung der heimischen Stammesmitglieder eine zentrale Rolle spielt. Angesichts der prognostizierten Bevölkerungsentwicklung ist ein Absinken des Bruttoinlandprodukts (BIP) pro Kopf der Bevölkerung von heute unter 400 US-Dollar zu erwarten, und das in einem Land, das heute bereits zu einem der ärmsten der Welt zählt. In dem Jahr von 2002 auf 2003 stieg die Zahl der unterhalb der Armutsgrenze lebenden Menschen von 23 auf 53 Prozent. Nach Angaben des Wirtschaftsministers stammen über 90 Prozent aller Waren auf dem afghanischen Markt aus Importen. ${ }^{38}$ Das ist Ausdruck einer nicht entwickelten Agrargesellschaft, die angesichts des Bevölkerungswachstums immer weniger in der Lage sein wird, den Menschen ein Auskommen zu bieten.

Politik: Im „failed state-Index“ 2007 steht Afghanistan an achtschlechtester Position. ${ }^{39}$ Freedom House bescheinigt dem als „halbfrei“ eingestuften Land einen Abwärtstrend bei den politischen und Freiheitsrechten. ${ }^{40}$ Es handelt sich um einen Rentierstaat, der von Vetternwirtschaft und Subsidien lebt. ${ }^{41}$ Die afghanische Regierung unter Präsident Karzai gilt als unfähig, korrupt und schwach, weil sie Personen in hohen Regierungsämtern akzeptieren muss, deren primäre Loyalität nicht der Regierung und dem Land gilt. Darüber hinaus werden 80 Prozent des Regierungsetats von der internationalen Gemeinschaft finanziert. ${ }^{42}$ Dass die Geberländer die Kontrolle über die

32 Siehe: CIA: The World Factbook (2008).

33 Baraki, Martin: Die Zerstörung Afghanistans, in: Zeit-Fragen, 13. August 2007, unter: http://www.antikriegsforum-heidelberg.de/afghan/hintergrund/zerstoerung afghanistans_baraki.html (Zugriff: 7. August.2008)

34 Siehe: Cordesman, Anthony H. (2008), S. 55.

35 Siehe: UNODC (2008).

36 Rashid, Ahmed: Taliban: Militant Islam, Oil and Fundamentalism in Central Asia, Yale: Yale University Press, 2008. Maley, William (Hrsg.): Fundamentalism Reborn? Afghanistan and the Taliban, New York: NYU Press, 1998.

37 Siehe: Schetter, Conrad (2007)

38 Siehe: Baraki, Martin (2007).

39 The Failed State Index 2007, Foreign Policy website: http://www.foreignpolicy. com/story/cms.php?story_id=3865.

40 Freedom in the World 2008, Freedom House website: http://www.freedomhouse.org/template.cfm?page $=395$.

41 Kühn, Florian P.: Supporting the State, depleting the State: Estranged state-societyrelations in Afghanistan, in: Ehrhart, Hans-Georg/Pentland, Charles (Eds.): The Afghanistan Challenge: Hard Realities and Strategic Choices, Montreal/Kingston: McGill-Queen's Press 2009 (in Vorbereitung).

42 Waldman, Matt: Aid Effectiveness in Afghanistan, Kabul: Agency Coordinating Body for Afghan Relief (ACBAR), Advocacy Series, März 2008, S. 6. 
Hilfsgelder im Bereich der Entwicklungspolitik behalten wollen, begründen sie mit der weit verbreiteten Korruption, allerdings sind Betrug, Missmanagement und Pfusch ${ }^{43}$ auch bei den internationalen Akteuren verbreitet. Die von außen geförderte zentrale Staatlichkeit steht in Konkurrenz zu den existierenden Strukturen einer Stammesgesellschaft. Deren einzelne Bestandteile sind einerseits autonom und lokal, andererseits sind sie über Arbeitsmigration mit der globalisierten Welt verbunden. Die Globalisierung stärkt also eher die alten lokalen Ordnungen als die von der internationalen Gemeinschaft angestrebte neue Staatlichkeit.

\section{Bewertung}

Die politische Zielsetzung: Das im Afghan Compact 2006 vorgegebene Ziel des Aufbaus einer demokratischen afghanischen Regierung, die das ganze Land regiert, wird vermutlich auf Dauer von einem Großteil der gesellschaftlichen Kräfte als eine Gefährdung ihrer Autonomie und ihrer traditionalen Ordnung wahrgenommen werden. Dies umso mehr, weil die Regierung Karzai als eine von Washington eingesetzte Marionettenregierung angesehen wird. Dies kommt nicht nur in der starken Fremdfinanzierung des Staatsetats und der Anwesenheit ausländischer Truppen zum Ausdruck, sondern auch darin, dass sie sich nur auf eine schmale Basis von westlich geprägten „Liberalen“ stützen kann, während das Parlament „increasingly became the stronghold of dissent, Afghan style, with a kalaidoscop of nationalist, ethnic, tribal and gender-based interests that were denied space to raise questions about sovereignty“. ${ }^{44}$ Dass die Regierung sehr viele jener Akteure, die von der afghanischen Bevölkerung als Kriegsverbrecher wahrgenommen werden, als Minister bestallt hat, schwächt sie weiter, ist doch deren Loyalität, soweit sie denn überhaupt existiert, schlichtweg erkauft. Die Zielsetzung einer Zentralregierung mit Kontrolle über die ländliche Peripherie wird in Afghanistan anhaltenden Widerstand hervorrufen, solange die Afghanen lediglich als Rezipienten eines für sie fremden Modells angesehen werden. Es ist langfristig möglich, dass sich andere politische Strukturen sozusagen von unten aus dieser Stammesgesellschaft entwickeln. Gleichwohl sind diese tribalen Strukturen heute die funktionsfähigsten im Lande. Der politische Aufbau Afghanistans sollte sie nutzen und den Menschen mehr Zeit für Entwicklung lassen. Dieser Prozess wird allerdings angesichts der gesellschaftlichen und wirtschaftlichen Entwicklungstrends nicht konfliktfrei verlaufen können.

Stabilität und Sicherheit: Die internationale Gemeinschaft hat sich vom proklamierten Ziel der Schaffung von Sicherheit als Voraussetzung für Stabilität und Entwicklung weiter entfernt. Über die letzten Jahre sind die gewaltsamen Aktivitäten des afghanischen Aufstands gewachsen. In den gleichen Zeitraum fällt die Ausdehnung von ISAF über das ganze Land. Der Begriff der Neo-Taliban verschleiert die wahren Ursachen des steigenden Gewaltpegels, die vor allem in anti-westlichen,

\footnotetext{
43 Nawa, Fariba: Afghanistan, Inc., Oakland: Corpwatch, A Corpwatch Investigative Report, 2006.

44 Tadibakhsh, Shahrbanou/Schoiswohl, Michael: Playing with Fire? The International Community's Democratization Experiment in Afghanistan, International Peacekeeping, Bd. 15, Nr. 2, 2008, S. 252-267, S. 257.
}

anti-modernen und anti-zentralistischen Haltungen vieler afghanischer Akteure liegen. Darüber hinaus wird durch die wachsende Drogenproduktion die politische Stabilität und die Sicherheit unterminiert. ${ }^{45}$ Dass die Entwaffnung von Stammesmilizen, Bürgerkriegsarmeen, Privatarmeen und anderen Akteuren gescheitert ist, hängt neben der Gewaltökonomie und der Kriegerkultur auch mit der Tatsache zusammen, dass die Stämme kein Vertrauen in die Sicherheitsorgane der Regierung in Kabul haben. Man sollte also darüber nachdenken, wie Sicherheit verstärkt durch regionale und lokale Akteure/Strukturen gewährleistet werden kann.

Wirtschaft und soziale Entwicklung: Das im Afghanistan Compact gesetzte Ziel eines nachhaltigen Wachstums zur Reduzierung von Hunger, Armut und Arbeitslosigkeit liegt noch in weiter Ferne. Die internationale Hilfe erhält die Regierung Karzai am Leben, bei der großen Mehrzahl der Bevölkerung sind die bisherigen Investitionen jedoch noch nicht angekommen. Die wirtschaftlichen Aktivitäten sind eher rückläufig, außer im kriminellen Sektor. Erfolge sind im Bildungssektor zu verzeichnen, sie wirken sich jedoch nicht kurzfristig aus und werden von den militant-islamischen Kräften hart bekämpft ${ }^{46}$. Das gesellschaftliche Gewaltpotenzial dürfte sich durch die zu erwartende anhaltend hohe Arbeitslosigkeit, die große Armut und die Perspektivlosigkeit in der nachwachsenden jungen Generation verstärken. Der Versuch, große Summen von Hilfsgeldern über den nicht funktionierenden Regierungsapparat der Regierung in Kabul verteilen zu wollen, muss zwangsläufig zu Korruption führen ${ }^{47}$. Die Entwicklungshilfe müsste also stärker und besser koordiniert in die lokalen Strukturen, vor allem unterhalb der Distriktsebene fließen. Zugleich sollte bedacht werden, dass nicht alle Staaten "are culturally capable and/or willing to accept the values of liberal market economics (...) especially those that essentially are 'honour-based' societies. ${ }^{48}$

Gesamtbewertung: Offensichtlich geht der strategische Ansatz der internationalen Gemeinschaft an der realen Situation in Afghanistan vorbei. Darüber hinaus ist zu vermuten, dass ein nicht unerheblicher Teil des Widerstands gerade auf diese Strategie zurückzuführen ist. ${ }^{49}$ Daher scheint es sinnvoll, eine gründliche Analyse der gesellschaftlichen, wirtschaftlichen und politischen Verhältnisse Afghanistans an den Anfang einer umfassenden Korrektur der strategischen Zielsetzung der internationalen Gemeinschaft zu stellen und davon das weitere Vorgehen abhängig zu machen. Vieles spricht dafür, den Menschen in Afghanistan mehr Autonomie (Afghan face) und Zeit (Afghan pace) für ihre weitere Entwicklung zu geben.

\section{Optionen}

Angesichts der vorstehenden Lageanalyse und Bewertung ergeben sich drei Handlungsoptionen.

45 Siehe: Roy (2004)

46 Siehe: Human Rights Watch (2006).

47 Siehe: Nawa (2006).

48 Pullinger, Stephen: European Security in 2020: Threats, Challenges and Responses, in: Brüssel: International Security Information Service, European Security Review, Nr. 37, S. 10-13, 2008, S. 12

49 Siehe: Tadibakhsh/Schoiswohl (2008), S. 263. 
1. Die erste Möglichkeit ist eine schnelle Beendigung des militärischen Engagements bei gleichzeitiger langfristiger Fortsetzung der entwicklungspolitischen Unterstützung Afghanistans. Die internationale Staatengemeinschaft reduziert ihre militärischen Aktivitäten graduell bis 2010 vollständig und legt die sicherheitspolitische Verantwortung in die Hände der afghanischen Regierung. Zugleich führt sie die zivilen Teile des bis Ende 2010 laufenden Afghanistan Compact bis 2020 weiter. Der Vorteil dieser Option liegt darin, dass er einen schnellen Rückzug der militärischen Kräfte ermöglichen würde, ohne das entwicklungspolitische Engagement aufzugeben. Der Nachteil wäre, dass ein kurzfristiger militärischer Rückzug der internationalen Gemeinschaft für das Land zu früh käme und von den meisten NATO-Partnern auch nicht akzeptiert würde. Dagegen spricht auch, dass eine bloße Fortführung des entwicklungspolitischen Ansatzes im Sinne eines „Weiter so“ nicht zielführend wäre, weil die bisherigen Ergebnisse auf diesem Gebiet insgesamt wenig erfolgreich waren.

2. Die zweite Option besteht darin, den bisherigen Ansatz zu verstärken und langfristig fortzusetzen. Er folgt der Devise, dass die sicherheits- und entwicklungspolitische Stabilisierung des Landes ein langfristiges Vorhaben ist, das ein nachhaltiges internationales Engagement in allen drei Aktionsbereichen - Sicherheit, Governance, Entwicklung - verlangt. Die aktive militärische Unterstützung wird angesichts der erstarkten Neo-Taliban und der unterentwickelten afghanischen Fähigkeiten mindestens noch weitere zehn Jahre fortgesetzt, die entwicklungspolitische wesentlich länger. Diese Option entspricht auf den ersten Blick der besten aller Welten. Doch was heißt das konkret? Wie viele Truppen sind ausreichend und wie viel Entwicklungshilfe kann das Land sinnvoll absorbieren? Die Umsetzung dieser Option würde einen wesentlichen Aspekt der afghanischen Souveränität und Eigenverantwortung („ownership“) auf lange Zeit blockieren. Eine signifikante Verstärkung des militärischen Einsatzes ist bereits aus Kosten- und politischen Erwägungen gegenwärtig nicht möglich. Seine langfristige Aufrechterhaltung wäre erst recht weder finanzierbar noch innenpolitisch durchsetzbar. Zudem ist nicht zu erwarten, dass ein verstärktes Engagement von außen den kulturellen Wandel herbeiführen kann, der notwenig wäre, um die afghanische Gesellschaft nach westlichem Vorbild zu ändern.

3. Die dritte Option ist ein umfassender Strategiewechsel, der fünf Aspekte beinhaltet:

- Bescheidenere Ziele: Die internationale Gemeinschaft nimmt davon Abstand, Afghanistan nach ihrem Ebenbild verändern zu wollen. Die im AC formulierten visionären Vorstellungen werden durch realistischere und somit glaubhaftere Ziele ersetzt. Es wird akzeptiert, dass angesichts der bisherigen Geschichte und des jetzigen Entwicklungsstandes Afghanistans, aber auch wegen begrenzter Mittel und Fähigkeiten der internationalen Geber keine perfekten oder visionären, sondern lediglich pragmatische und erreichbare Ziele angestrebt werden können. ${ }^{50}$

50 Etzioni, Amitai: Weniger ist mehr, in: Frankfurter Allgemeine Zeitung, 28. Juli 2008, S. 8.
- Afghanisierung der Sicherheit: Da die Aufstandsbekämpfung in Afghanistan militärisch mittelfristig nicht und langfristig - wenn überhaupt - nur zu einem unakzeptablen Preis zu gewinnen ist, fährt die internationale Staatengemeinschaft ihre Kampfeinsätze zurück und beendet diese nach drei bis fünf Jahren. Danach werden die ANA und die ANP für die Sicherheit des Landes alleine verantwortlich sein. Zugleich wird wesentlich mehr als bislang in die Reform der Sicherheitssektoren investiert, vor allem in die Reform der Polizei und die sie führenden lokalen, regionalen und nationalen Administrationen. Die Festlegung eines Datums (end date) für den Abzug des größten Teils der NATOTruppen hat den Vorteil, der souveränen Regierung einen klaren Übergangszeitraum zu kommunizieren und zugleich ihre Eigenverantwortlichkeit zu stärken, um zu tragfähigen Arrangements mit der lokalen Ebene zu kommen. Da es sich bei der Sicherheitssektorreform um einen langfristigen Prozess handelt, unterstützt die internationale Staatengemeinschaft Afghanistan in diesem Bereich weiter. ${ }^{51}$

- Dezentraler Governance-Ansatz: Der bislang verfolgte Versuch, zentralstaatliche Regierungsstrukturen aufzubauen, wird ergänzt durch die nachhaltige Förderung lokaler Strukturen. Die gesamte Befriedungs- und Wiederaufbaustrategie trägt der historisch-kulturellen Tatsache Rechnung, dass sich zentralistische Ansätze in diesem Land noch nie haben durchsetzen können und von lokalen Akteuren als Bedrohung angesehen werden. Da in Stammesgesellschaften die primäre Loyalität der Menschen nicht dem Staat gehören kann, werden dezentrale Governance-Ansätze gestärkt. Es wird akzeptiert, dass es sich dabei um fluide Machtarrangements handelt, die primär lokalen Interessen dienen und deren Loyalität gegenüber der Zentralregierung relativ schwach bleibt.

- Angepasste Entwicklungsstrategie: Das Ziel des Aufbaus einer liberalen Marktwirtschaft wird relativiert, die entwicklungspolitische Rolle des Staates auf allen Ebenen gefördert. Lokale Akteure werden gestärkt, lokale Bedürfnisse in den Vordergrund der Aktivitäten gestellt. Dabei wäre ein Kontext zwischen der Vergabe von entwicklungspolitischen Mitteln und der Gewährleistung von Sicherheit durch die lokalen Akteure eine sinnvolle Bedingung, um Sicherheit und Entwicklung zu fördern. Die Drogenbekämpfung erfolgt durch das Angebot alternativer Produktions- und Absatzmöglichkeiten. Infrastrukturmaßnahmen, Bildung und Ausbildung werden insbesondere im ländlichen Raum gefördert.

- Regionale Einbettung: Der Afghanistankonflikt ist nur im regionalen Rahmenzu lösen. ${ }^{52}$ Folglich werden die Nachbarstaaten, insbesondere Pakistan und Iran, und andere strategisch relevante Akteure wie Indien oder Russland sowie andere interessierte Akteure bei seiner Bewältigung eingebunden. Eine Regionalkonferenz

51 Ehrhart, Hans-Georg/Schnabel, Albrecht: Post-Conflict Societies and the Military: Recommendations for Security Sector Reform, in: Schnabel/Albrecht/Ehrhart, Hans-Georg, (Hg.): Security Sector Reform and Post-Conflict Peacebuilding, Tokyo, New York, Paris: United Nations University Press 2005, S. 315-322.

52 Wagner, Christian: Pakistan Policy towards Afghanistan in the Shadow of India, in: Ehrhart / Pentland (2009). 
über Sicherheit, wirtschaftliche Zusammenarbeit und Entwicklung könnte den Auftakt für einen politischen Prozess darstellen, der zu einem alle Bereiche abdeckenden Grundlagenabkommen, zu Aktionsplänen und anschließenden Überprüfungskonferenzen führt. Kernelemente dieses Vertrags wären ein Gewaltverzicht und die internationale Garantie für eine immerwährende Neutralität Afghanistans.

Gegen diese Option spricht, dass sie von der internationalen Staatengemeinschaft ein radikales Umdenken verlangt, das angesichts der vorherrschenden beschönigenden und realitätsfernen Lageanalyse kaum zu erwarten ist. Dafür dürfte sprechen, dass jede Form der Hybris früher oder später zu Kursänderungen und Strategiewechseln führt.

\section{Schlussfolgerung}

Die dritte Möglichkeit enthält die meisten Handlungsoptionen und bietet auch im Sinne des entwicklungspolitischen
Grundsatzes von „do no harm“ mehr Möglichkeiten, Gewalteskalation zu vermeiden. Wesentliche Gründe dafür sind oben aufgeführt. Die zentralen Faktoren, warum die heutige Strategie des Westens wahrscheinlich scheitern wird, sind kulturelle Differenz, begrenzte Ressourcen und die Unmöglichkeit, das Sozialverhalten einer ganzen Gesellschaft von außen grundlegend zu verändern. Die große Gefahr ist, dass aus Enttäuschung eine doppelte Gegenreaktion hervorgerufen werden könnte. Die afghanische Gesellschaft könnte die internationale Gemeinschaft zunehmend als Besatzer wahrnehmen und die internationale Gebergemeinschaft könnte sich mehr oder weniger abrupt von Afghanistan abwenden. Folglich ist ein umfassender Strategiewechsel notwendig, der fünf Aspekte beherzigt: bescheidenere Ziele, Afghanisierung der Sicherheit, dezentrale Regierungsstrukturen, auf lokale Bedürfnisse ausgerichtete Entwicklung und regionale Einbettung des Afghanistankonflikts.

\title{
NGO Views of NATO Strategy in Afghanistan
}

\author{
Lara Olson and Anja de Beer*
}

\begin{abstract}
NGOs in Afghanistan have long argued that a fundamental flaw of the NATO effort is the extensive involvement of the military in the civilian roles of reconstruction and development. This has undermined humanitarian space and access in Afghanistan, arguably undermining prospects for the overall development effort. NGO advocacy inside Afghanistan and in donor countries has consistently argued for the NATO strategy to address this counterproductive militarization of aid, as well as the need for clear civilian leadership and a unified strategy across all members of the ISAF mission. Furthermore, for the best possible strategies for Afghan recovery to emerge, the views of the NGO sector must be given more than a token role in the policy dialogue with NATO.
\end{abstract}

Keywords: NGOs, Afghanistan, NATO, humanitarian organisations, NROs, Afghanistan, NATO, humanitäre Organisationen

\section{Introduction}

I nternational and Afghan non-governmental organizations (NGOs) constitute a major resource for recovery in Afghanistan given their direct work with Afghan communities, their development expertise, and their major operational role as partners of the Government of Afghanistan (GOA), the United Nations Mission in Afghanistan (UNAMA), and international donors. However, NGOs have faced a very difficult environment in which to make their voices heard. Unlike UN peacekeeping contexts, the international effort in Afghanistan lacks strong civilian leadership and for the last years has been

\footnotetext{
* Lara Olson is Co-Director, Peacebuilding, Development and Security Program, Centre for Military and Strategic Studies, University of Calgary, Canada; Anja de Beer is Director, Agency Coordinating Body for Afghan Relief (ACBAR), Kabul, Afghanistan.
}

led by the US and NATO military mission - by default. Furthermore, 7 years after the fall of the Taliban, Afghanistan is still not a post-conflict country. There continues to be a volatile mixed war and peace context where counterinsurgency strategies, an expansive statebuilding agenda, and reconstruction, relief and development efforts coexist and sometimes collide. Furthermore, the marked deterioration in security in many parts of the country over the past year has given urgency to calls for greater, stronger, tighter coordination of all international efforts.

Today's focus on better coordination and integration within the international effort implies that the fundamental strategies are sound and only the execution is faulty. In contrast, operational humanitarian and development NGOs in Afghanistan have argued continuously for a rethink of some fundamental tenets of the international mission - especially its approach to security and the deep conflation of civilian and military roles in relief, reconstruction and development. 